\title{
Author Correction: Emergence of the topological Hall effect in a tetragonal compensated ferrimagnet $\mathrm{Mn}_{2.3} \mathrm{Pd}_{0.7} \mathrm{Ga}$
}

Won-Young Choi, Woosuk Yoo and Myung-Hwa Jung (D)

Correction to: NPG Asia Materials

https://doi.org/10.1038/s41427-021-00347-3

published online 24 December 2021

In the sentence (page 5) beginning 'by using the relation...' in this article, the equation ' $\Delta S_{M}=\frac{1}{\Delta T}\left[\int_{0}^{H}\left(M_{1}\right) d H \int_{0}^{H}\left(M_{2}\right) d H\right]$ ' should have read ' $\Delta S_{M}=\frac{1}{\Delta T}\left[\int_{0}^{H}\left(M_{1}\right) d H-\int_{0}^{H}\left(M_{2}\right) d H\right]$ '.
The original article has been corrected.

Published online: 22 February 2022 